\title{
Effects of Gas Area Fraction on Developing Flow of Shear Thickening Fluids in Circular Tube having Superhydrophobic Transverse Grooves
}

\author{
Han Wei Lee ${ }^{1}$, Kok Hwa Yu ${ }^{1,}{ }^{*}$, Yew Heng Teoh ${ }^{1}$, Mohd Azmi Ismail ${ }^{1}$ \\ 1 School of Mechanical Engineering, Engineering Campus, Universiti Sains Malaysia, 14300 Penang, Malaysia
}

\section{ARTICLE INFO}

\section{Article history:}

Received 19 March 2020

Received in revised form 18 May 2020

Accepted 23 May 2020

Available online 31 May 2020

Keywords:

Water repellent; non-Newtonian; surface roughness; air cavity; power-law index

\section{ABSTRACT}

Superhydrophobic surface is extensively used in lab-on-a-chip technology, thermal management, self-cleaning and condensation arising from its ability to reduce fluid flow resistance. There are many existing studies conducted on fluid flow in channels and tubes having superhydrophobic surface, especially focusing on Newtonian fluid in fully-developed flow region. Before a fully-developed flow profile is attained, fluid flow undergoes a developing velocity profile region where velocity flow profile continuously develops axially. However, limited studies are focused on flow developing region, especially non-Newtonian flow in the presence of superhydrophobic surface. In this study, the effects of gas area fraction on hydrodynamic entrance region of shear thickening fluids in a circular tube with alternating superhydrophobic grooves and ribs arranged in the transverse direction are investigated. Superhydrophobic transverse grooves of normalized groove-rib periodic spacing $L=0.1$ with different gas area fractions $\delta$ are considered in this study with the assumption of laminar, steady and incompressible flow in the tube. The influence of the superhydrophobic surface on the velocity field, centerline velocity distribution and hydrodynamic entrance length are examined. From the numerical results, for Reynold number, $R e$ of $1 \times 10^{-4}$ and power-law index, $n$ ranging from 1.0 to 1.5, the hydrodynamic entrance length is found to be consistently longer, in the presence of superhydrophobic surface. For microfluidic applications, this implies that for shear thickening flow through tube patterned with these regular microstructures of hydrophobic condition, flow developing region can be altered.

Copyright @ 2020 PENERBIT AKADEMIA BARU - All rights reserved

\section{Introduction}

Superhydrophobic surfaces are water-repellent surface patterned with microstructures that repel liquid from wetting its surface. The water-repellent surfaces exist naturally which can be observed on the lotus leaves. There are a lot of studies on the application of these surfaces [1]. The prime benefit of water-repellent surface is its ability in reducing the flow resistance, thereby it can

\footnotetext{
* Corresponding author.

E-mail address: yukokhwa@usm.my (Kok Hwa Yu)
}

https://doi.org/10.37934/cfdl.12.5.101110 
be employed in gaining higher mass flow rate in narrow tube such as microtube when the pumping power is limited [2-5]. Benefits arising from these surfaces can be applied in many applications such as Lab-on-chip technology [6], thermal management [7,8], self-cleaning [9], condensation [10], etc. Most of the recent studies on superhydrophobic surface in channels or tubes is limited to hydrodynamic fully-developed regions $[4,5,11]$. Although there are some exceptions where recent studies on superhydrophobic are focused on hydrodynamic developing regions [12-20].

In the absence of superhydrophobic surface, when Newtonian fluid flows in a tube having smooth wall, the dimensionless hydrodynamic entrance length is found to vary nonlinearly with the Reynolds number as predicted by Durst et al., [21]. With the presence of superhydrophobic surface, the hydrodynamic entrance length is predicted to be slightly longer than that of smooth walls $[18,19]$. In real life applications such as in chemical and processing industries, non-Newtonian fluid is often used instead of Newtonian fluid. The behavior of non-Newtonian fluid can be modeled via power-law fluid model. Using this model, the shear rate of the fluid varies using the power-law index (n), i.e., represents shear thinning fluid when $n<1$ and represents shear thickening fluid when $n>1$. For non-Newtonian fluid flow in circular tube with smooth wall, the dimensionless hydrodynamic entrance length can be approximated using correlation presented by Poole and Ridley [22], given by

$\frac{L_{h}}{D}=\left[\left(0.246 n^{2}-0.675 n+1.03\right)^{1.6}+(0.0567 R e)^{1.6}\right]^{1 / 1.6}$

where $D$ is the tube diameter, $L_{h}$ is the hydrodynamic entrance length and $R e$ is defined as [22]

$R e=\frac{\rho U_{a v g}^{2-n} D^{n}}{k} 8\left(\frac{n}{6 n+2}\right)^{n}$

where $U_{\text {avg }}$ is the average fluid velocity, $\rho$ is the density of the fluid and $k$ is the consistency index. It is worth to note that the correlation stated in Eq. (1) is valid for power-law index ranging $0.4<n<$ 1.5 and Reynolds number in the range of $0<R e<1000$. A similar research is also conducted on the plane channel flow where the hydrodynamic entrance length is also influenced by power-law index. The study of Haase et al., [14] focused on non-Newtonian fluid flow over surface with slip surface. This slip surface can be modelled either using wall slip [16] or textured surface $[14,19,20]$ (i.e., superhydrophobic transverse groove). A rough estimation of the hydrodynamic entrance length is proposed by Haase et al., [14] that factor in the influences of gas area fraction and the normalized groove-rib periodic spacing. Meanwhile, full numerical simulation in investigating the hydrodynamic entrance length for non-Newtonian fluid flow in microtube with superhydrophobic grooves was thus performed by Lee et al., [20] recently. The work of Lee et al., [20] shows the hydrodynamic entrance length is influenced by Reynolds number and power-law index.

In an internal flow, before a fully developed condition is achieved, the developing flow shall first prevail. Although there are some preliminary studies performed recently pertaining to slip and superhydrophobic walls on developing flow region, the effect of gas area fraction in the hydrodynamic developing region has not been systematically explored, especially for shear thickening fluid. It is important to characterize the shear thickening fluid behavior and gain insights on the alteration of the superhydrophobic surface scale on hydrodynamic entrance length.

\section{Methodology}

Figure 1 depicts illustration of fluid flow in circular tube with alternating superhydrophobic grooves and ribs. The groove-rib unit length is denoted by $E$ and the width of the groove is $e$. The 
scale of the superhydrophobic structures are governed by the dimensionless gas area fraction $(\delta=$ $e / E)$ and normalized groove-rib periodic spacing $(L=E / D)$. The tube has the diameter of $D$ with the circular coordinate system $(r, \theta, z)$. The axial and radial directions are represented by $z-$ and $r$-direction, respectively. While $\theta$ represents the azimuthal direction. However, the velocity of the fluid flow is only considered in $z$-direction. No flow is assumed in the $r$ - and $\theta$-direction. Steady laminar flow with incompressible power-law fluid is considered in this study. The superhydrophobic surface is represented by alternating groove and ribs surface. No-slip boundary and shear-free conditions are employed to mimic the solid-liquid (on the ribs) and liquid-gas interface (over the grooves), respectively. The net mass flow rate through the gas layer trapped in the grooves is assumed to be zero.

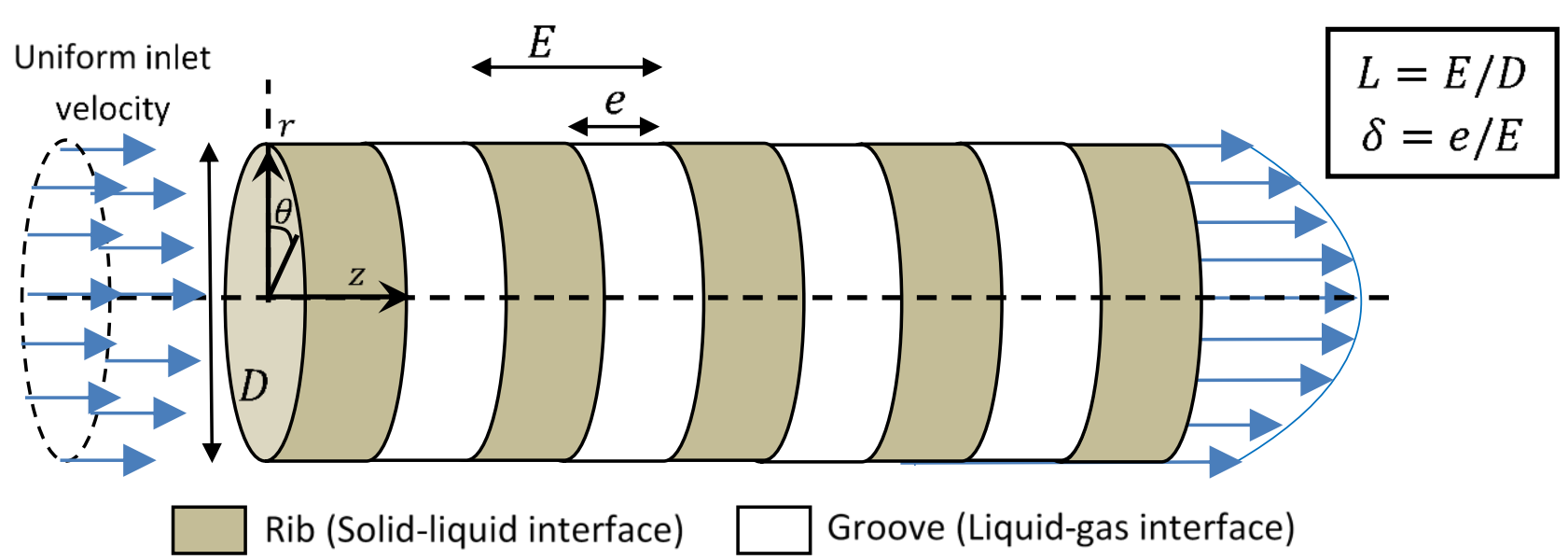

Fig. 1. Schematic diagram depicting the velocity profile for flow in tube having alternating superhydrophobic grooves and ribs

The governing equations in this study are stated as follow

$\frac{1}{r} \frac{\partial}{\partial r}\left(r u_{r}\right)+\frac{\partial u_{z}}{\partial z}=0$

$u_{r} \frac{\partial u_{r}}{\partial r}+u_{z} \frac{\partial u_{r}}{\partial z}=-\frac{1}{\rho} \frac{\partial p}{\partial r}+\frac{1}{r \rho} \frac{\partial\left(r \tau_{r r}\right)}{\partial r}+\frac{1}{\rho} \frac{\partial \tau_{z r}}{\partial x}-\frac{\tau_{\theta \theta}}{r \rho}$

$u_{r} \frac{\partial u_{z}}{\partial r}+u_{z} \frac{\partial u_{z}}{\partial z}=-\frac{1}{\rho} \frac{\partial p}{\partial z}+\frac{1}{\rho} \frac{\partial \tau_{z Z}}{\partial z}+\frac{1}{r p} \frac{\partial\left(r \tau_{r z}\right)}{\partial r}$

where $\tau_{r r}, \tau_{z r}, \tau_{r z}, \tau_{\theta \theta}$ and $\tau_{z z}$ are viscous stress components, given by

$$
\begin{aligned}
& \tau_{r r}=\eta\left(2 \frac{\partial u_{r}}{\partial r}\right) \\
& \tau_{\theta \theta}=\eta\left(2\left(\frac{1}{r} \frac{\partial u_{\theta}}{\partial \theta}+\frac{u_{r}}{r}\right)\right) \\
& \tau_{z z}=\eta\left(2 \frac{\partial u_{z}}{\partial z}\right) \\
& \tau_{r z}=\tau_{z r}=\eta\left(\frac{\partial u_{r}}{\partial z}+\frac{\partial u_{z}}{\partial r}\right)
\end{aligned}
$$


The $\eta$ denotes the power-law generalized Newtonian fluid (GNF) viscosity function, $\eta(\gamma)=\dot{k} \dot{\gamma}^{n-1}$ where $k$ is the consistency index, $\dot{\gamma}$ is the rate of deformation and $n$ is called the power-law index. It is worth to mention again that for $n<1$, the fluid is shear thinning fluid while $n>1$ is shear thickening fluid. Meanwhile, for $n=1$, it is a Newtonian fluid.

For fluid flow in microtube having superhydrophobic transverse grooves, the flow is independent of $\theta$-direction. Thus, the three-dimensional flow problem can be reduced to two-dimensional axisymmetric flow problem. Commercial computational fluid dynamics (CFD) software package ANSYS FLUENT 19.2 is used to solve the governing equations via finite volume method. The pressurevelocity coupling was solved via SIMPLE scheme. The numerical solution for pressure and momentum are based on second-order and second-order upwind schemes, respectively. The convergence criteria with scaled residuals of $10^{-10}$ for continuity and momentum equations are employed in this study. At the inlet uniform velocity, $U_{\text {inlet }}$ is applied while zero static pressure is applied at the outlet.

\section{Results and Discussion}

In this study, shear thickening fluid flowing at Reynolds number $R e=0.001$ in a tube having superhydrophobic transverse grooves of $L=0.1$ is considered. Along the superhydrophobic surface, the liquid-gas interfaces are assumed to be perfectly flat. In the simulation, a sufficiently long tube $(10 D)$ is employed to allow flow to reach fully-developed flow condition. The range of power-law index $(n)$ between 1 and 1.5 is considered. The consistency index $(k)$ is fixed to be 1 . Grid independence test was first conducted using four different grid resolutions (i.e., $N_{z} \times N_{r}=400 \times$ $10,800 \times 20,1600 \times 40$ and $3200 \times 80$ ) on smooth wall for power-law index of 1 . As can be seen from Figure 2, the resulted normalized hydrodynamic entrance length, $L_{h} / D$ converged when finer grid resolution is employed. Comparing the grid resolution of $N_{z} \times N_{r}=1600 \times 40$ and that of $N_{z} \times$ $N_{r}=3200 \times 80$, the deviation is less than $0.02 \%$.

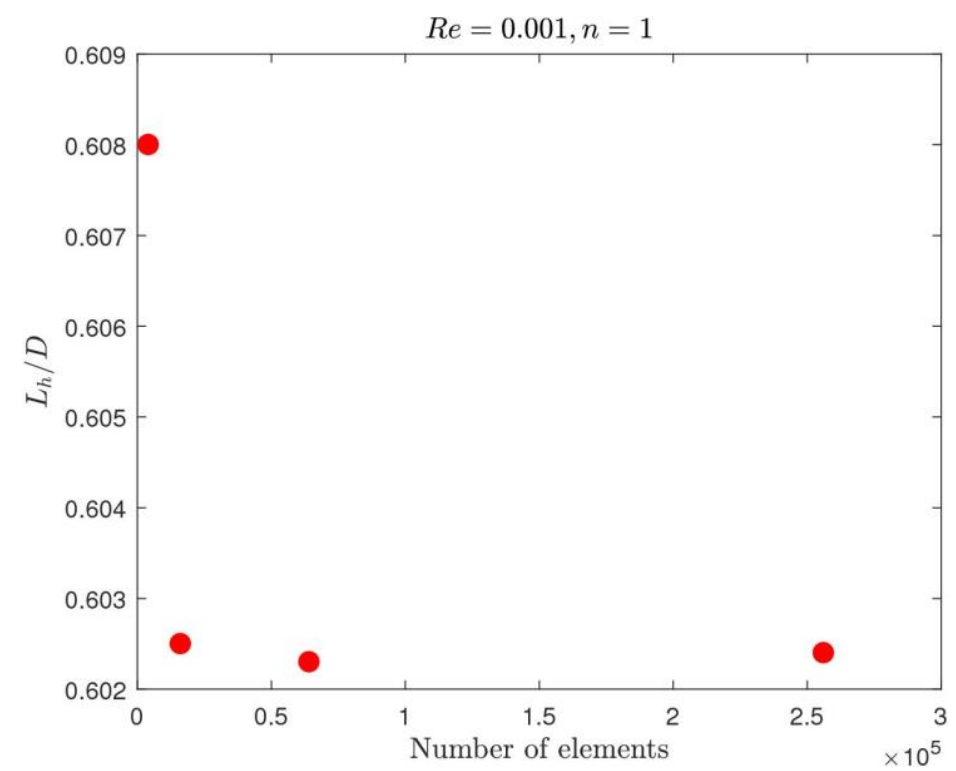

Fig. 2. Grid independence test for flow through tube having smooth wall at $R e=0.001$ for $n=1$

Using a fine grid resolution $\left(N_{z} \times N_{r}=3200 \times 80\right)$, the non-Newtonian flow problem is thus simulated and validated with existing data. Based on the grid resolution used, the non-dimensional grid spacing of $\Delta z / D=0.003125$ and $\Delta r / D=0.00625$ are consistently employed throughout this 
study. As showed in Figure 3, at $R e=0.001$, the numerical results yielded for flow in a tube having smooth wall are in good agreement with the correlation presented by Poole and Ridley [22], given in Eq. (1). The deviations between both are less than $2 \%$.

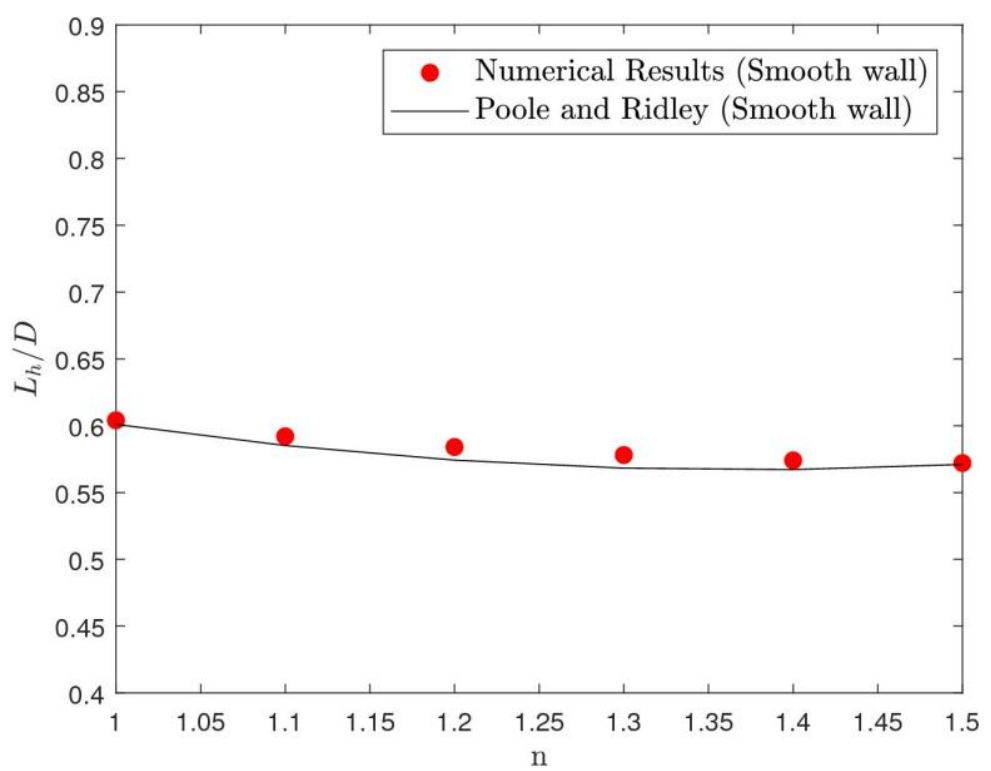

Fig. 3. Validation for normalized hydrodynamic entrance length, $L_{h} / D$ against power-law index for shear thickening fluid

\subsection{Effect on Velocity Field}

The influences of gas area fraction are first investigated on the velocity distribution over the tube. Three different gas area fractions were studied, i.e., $\delta=0.25,0.50$ and 0.75 . Gas area fraction represents the ratio of area occupied by liquid-gas interface over a groove-rib period. A smaller value of $\delta$ indicates that the area of liquid-gas interface is relatively smaller and vice versa. For $\delta=0.5$, the areas for liquid-gas interface and solid-liquid interface are equal.

As shown in Figure 4 for $n=1.3$, the normalized axial velocity in the developing region changes when different gas area fractions are employed. The main contrast is visible at the vicinity of the superhydrophobic wall. The increase in the gas area fraction will allow higher flow rate in the region close to the superhydrophobic wall, which is also observed in the fully-developed region [5]. To maintain the same flow rate through the tube, the redistribution of the fluid flow caused the fluid flow around the centerline to be lower when a large gas area fraction is employed. For $n=1.3$, the normalized velocity along the centerline can exceed twice the value of inlet velocity when $\delta=0.25$, as illustrate in Figure 4(a). However, the normalized maximum velocity in the bulk region is observed to be lower when a larger gas area fraction is employed. As can be observed in Figure 4(c), when gas area fraction is of 0.75 , the maximum normalized velocity along centerline is reduced to less than twice the inlet velocity. It is also worth to note that when a larger value of $\delta$ is employed, the velocity field in the vicinity of the superhydrophobic wall is significantly influenced by the surface microfeatures as can be seen in Figure 4(c). It is visible in this figure that the velocity field exhibits periodic pattern in the region where $r / D>0.4$. When $\delta$ is smaller, the effects of the superhydrophobic wall on velocity distribution deemed to dwindle. 


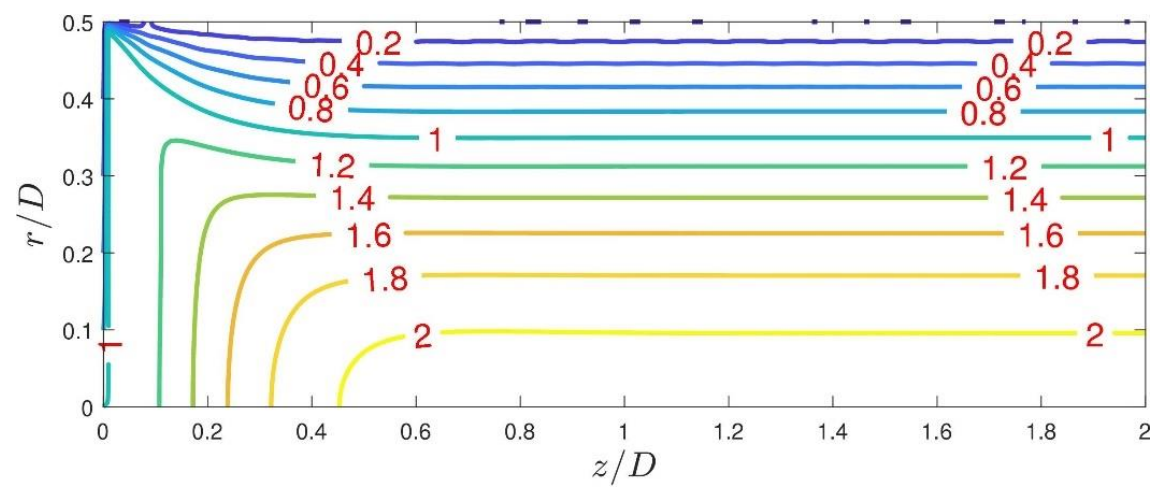

(a)

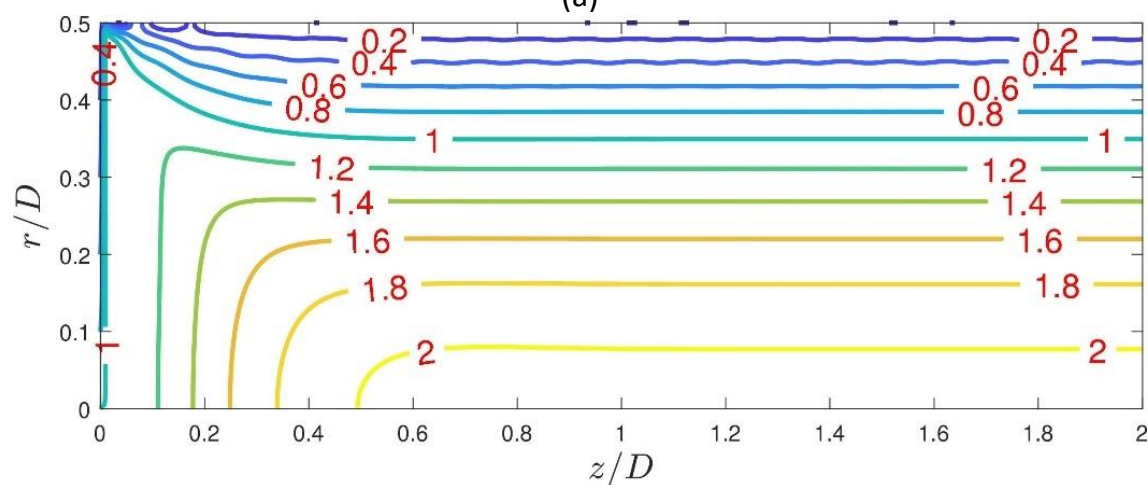

(b)

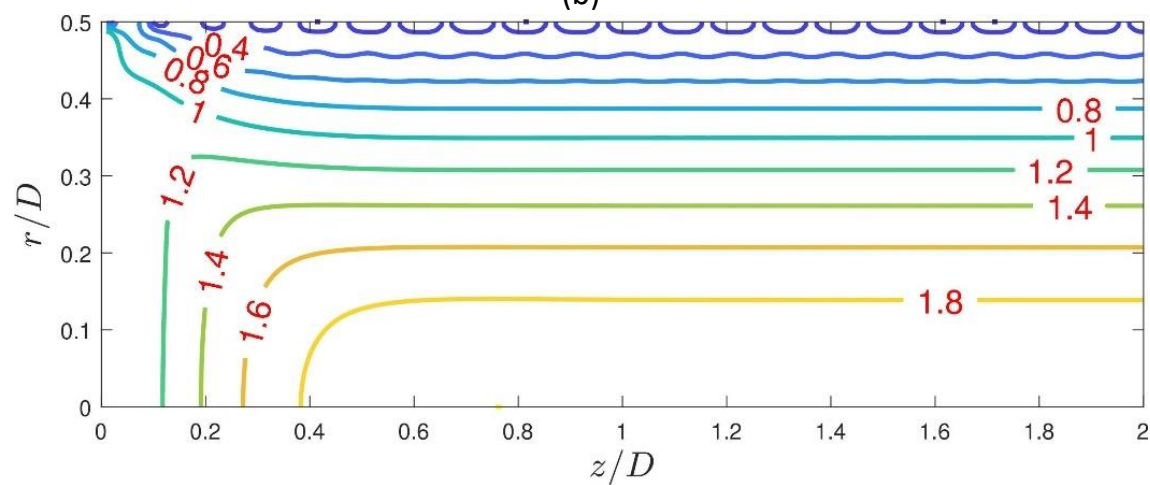

(c)

Fig. 4. Contours of normalized velocity field $\left(u_{z} / U_{\text {inlet }}\right)$ for non-Newtonian fluid with $n=1.3$ past superhydrophobic grooves of (a) $\delta=0.25$, (b) $\delta=$ 0.50 and (c) $\delta=0.75$

\subsection{Effect on Velocity Distribution Along Centerline}

Based on the normalized velocity fields, the velocity distributions along centerline are then plotted (as depicted in Figure 5) for all three gas area fractions. It is also worth to highlight that the normalized velocity distribution along tube centerline rises monotonically from the inlet until it reached fully-developed condition, similar trend with that of smooth surface. At $\delta=0.25$, maximum normalized velocity is at 2.113. The maximum normalized velocity reduced to 2.074 and 1.994 when $\delta=0.5$ and $\delta=0.75$, respectively. For all three gas area fractions investigated, the velocity distribution along the centerline is deemed not affected by the superhydrophobic wall structures. No periodic pattern is observed on velocity field along the centerline of the fluid flow. This is mainly due to the small value of normalized groove-rib periodic spacing being used (i.e., $L=0.1$ ) where the surface structures are relatively smaller than the size of the tube. 


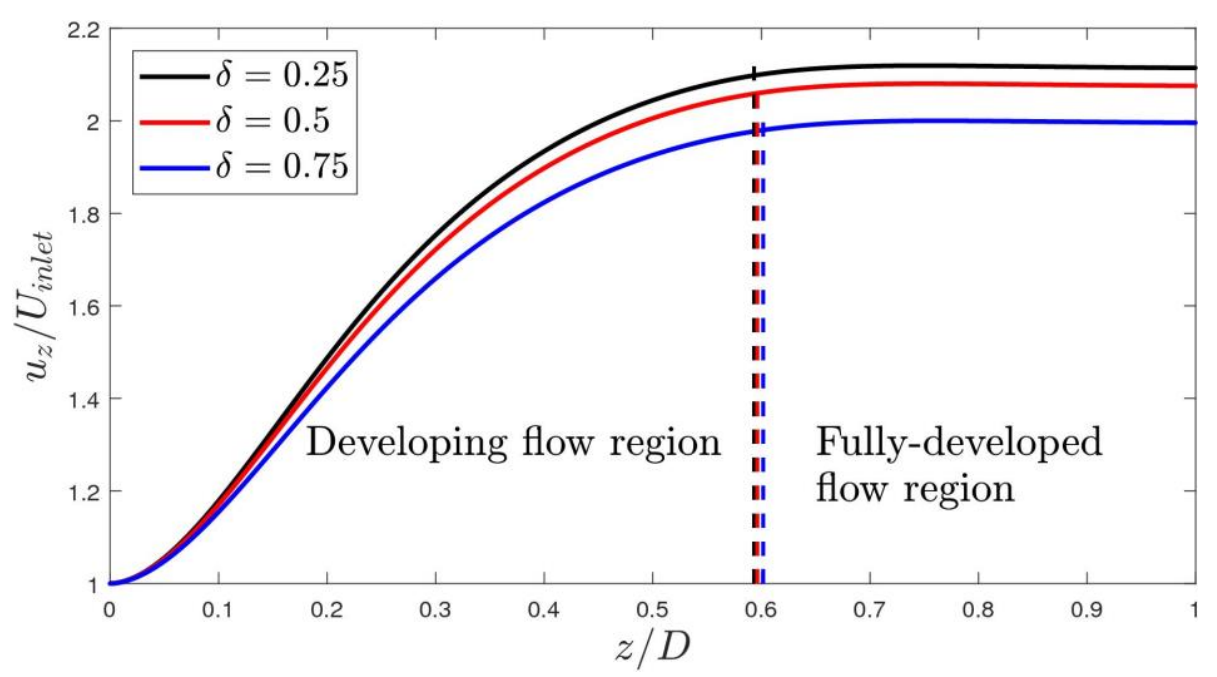

Fig. 5. Normalized axial velocity along tube centerline for power law fluid $n=$ 1.3 for superhydrophobic transverse grooves of $\delta=0.25,0.50$ and 0.75

\subsection{Effect on Hydrodynamic Entrance Length}

Depicted in Figure 6 , it is visible that the normalized hydrodynamic entrance length, $L_{h} / D$ increases as the gas area fraction $\delta$ increases. The present numerical simulation for $n=1.3$ shows that $L_{h} / D=0.5934$ when $\delta=0.25$ (given that $L=0.1$ ). $L_{h} / D$ increases to 0.5962 and 0.6018 when $\delta=0.5$ and $\delta=0.75$ are employed, respectively. It is worthwhile to note that the hydrodynamic entrance length calculated is the length required for centreline velocity to reach $99 \%$ of the fully-developed velocity in the axial direction. The increase in the normalized hydrodynamic entrance length when a larger gas area fraction is employed may be arising from the weaken inertial force at the bulk of the fluid flow. With a larger gas area fraction, the redistribution of the fluid flow with more flow is allowed close to the superhydrophobic wall, thereby reducing the inertial force in the bulk of the flow. This then creates a delaying effect that exhibits a longer length for fluid to reach fully-developed condition.

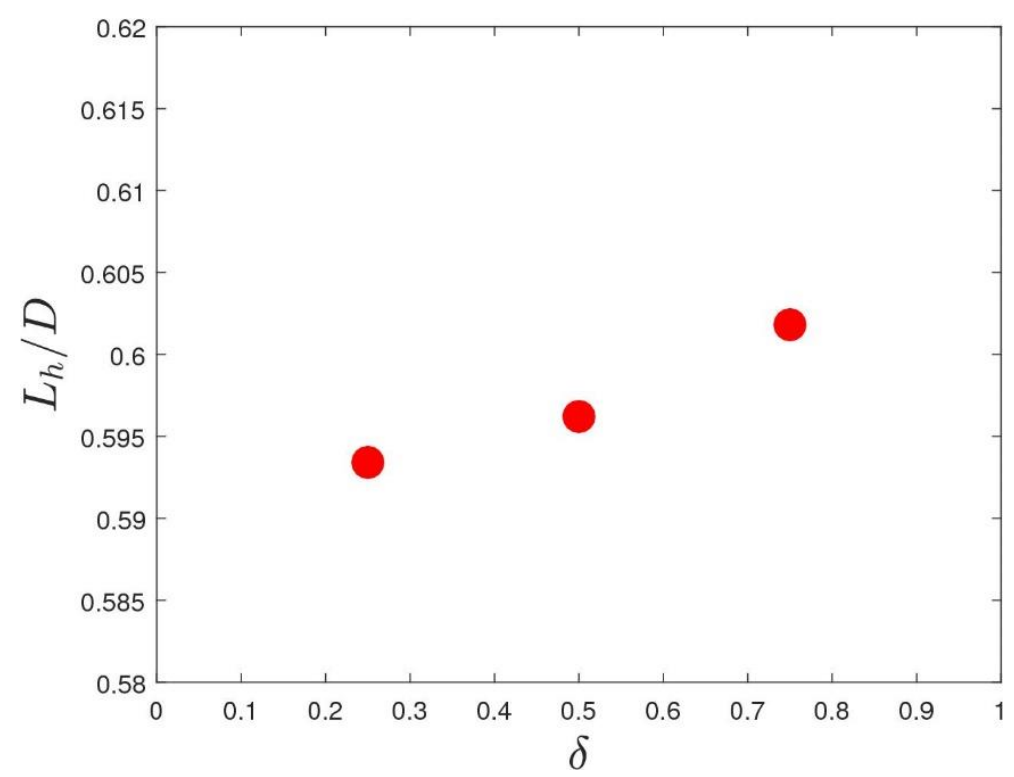

Fig. 6. Variation of normalized hydrodynamic entrance length, $L_{h} / D$ against gas area fraction, $\delta$ of $0.25,0.5$ and 0.75 for nonNewtonian fluid flow of $n=1.3$ 


\subsection{Effect of Power-Law Index on Hydrodynamic Entrance Length}

Apart from the results devoted to $n=1.3$ presented earlier, the effects of varying power-law index on hydrodynamic entrance length are also studied. As can be observed from Figure 7, the presence of superhydrophobic transverse grooves consistently yield a longer $L_{h} / D$. However, the trends are visibly different between Newtonian fluid $(n=1)$ and shear thickening fluid $(n>1)$. For Newtonian fluid, a small fraction of superhydrophobic transverse grooves will yield a small increase in $L_{h} / D$. Increase in $L_{h} / D$ rises rapidly with the increase in $\delta$. In contrast, an opposite trend is observed for large $n$ (i.e., $n=1.5$ ). Patterning a small fraction of superhydrophobic transverse grooves could significantly extend the hydrodynamic entrance length. However, further increase in gas area fraction doesn't affect the hydrodynamic entrance length significantly. For shear thickening fluid, the apparent viscosity at the wall is higher as compared to that of bulk region. Therefore, the introduction of shear-free region along the wall will allow relatively high flow rate to pass through in the region close to the wall. This causes the significant influence that yield a much longer $L_{h} / D$ when $n=1.5$ is employed.

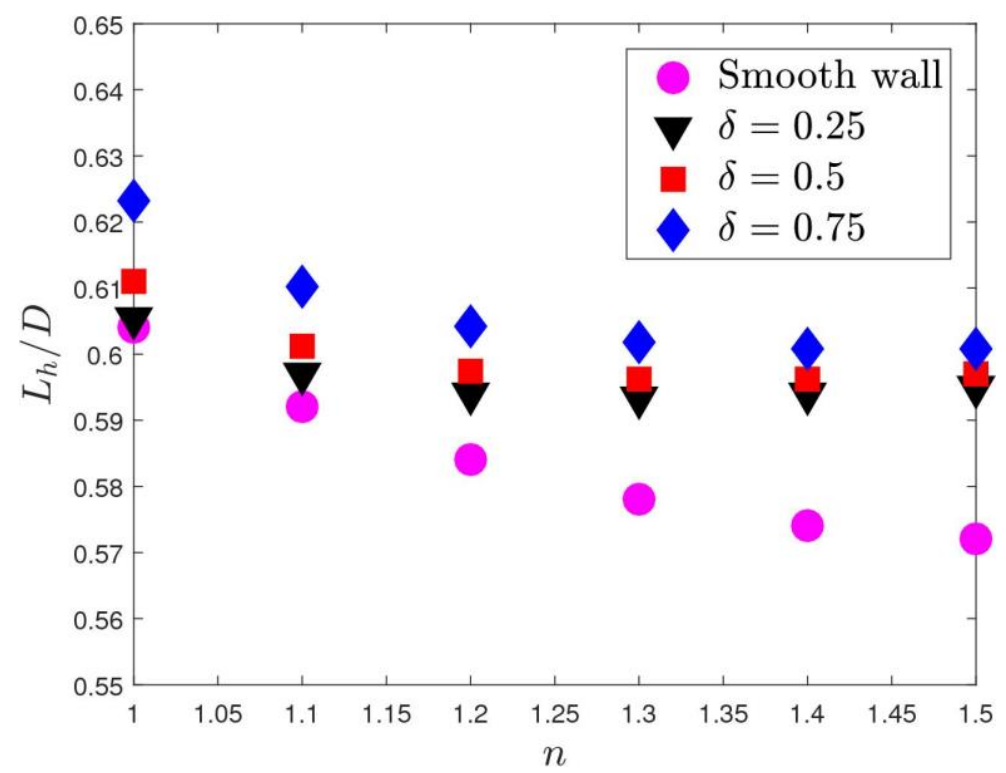

Fig. 7. Variation of the normalized hydrodynamic entrance length, $L_{h} / D$ against power-law index, $n$ for fluid flow in tube having different gas area fraction $\delta=0,0.25,0.5$ and 0.75 . Smooth wall is indicated by $\delta=0$

\section{Conclusion}

Numerical simulations were employed to investigate the developing flow of shear thickening fluids in tube have superhydrophobic transverse grooves. At low Reynolds number ( $R e=$ 0.001), normalized hydrodynamic entrance length $\left(L_{h} / D\right)$ is consistently higher when superhydrophobic transverse grooves are present. For shear thickening fluid with high value of $n$, the influence on $L_{h} / D$ is dominated by the presence of this salient surface. Meanwhile, the increase in $L_{h} / D$ for Newtonian fluid $(n=1)$ is largely influenced by the size of the superhydrophobic grooves. 


\section{Acknowledgement}

The authors acknowledge the Ministry of Education (MOE) of Malaysia under Fundamental Research Grant Scheme (FRGS Grant: FRGS/1/2019/TK03/USM/03/3) and School of Mechanical Engineering for the financial support.

\section{Reference}

[1] Law, Kock-Yee. "Definitions for Hydrophilicity, Hydrophobicity, and Superhydrophobicity: Getting the Basics Right." The Journal of Physical Chemistry Letters 5, no. 4 (2014): 686-688. https://doi.org/10.1021/jz402762h

[2] Lauga, Eric, and Howard A. Stone. "Effective slip in pressure-driven Stokes flow." Journal of Fluid Mechanics 489, no. (2003): 55-77. https://doi.org/10.1017/S0022112003004695

[3] Ou, Jia, Blair Perot, and Jonathan P. Rothstein. "Laminar drag reduction in microchannels using ultrahydrophobic surfaces." Physics of Fluids 16, no. 12 (2004): 4635-4643. https://doi.org/10.1063/1.1812011

[4] Davies, J., D. Maynes, B. W. Webb, and B. Woolford. "Laminar flow in a microchannel with superhydrophobic walls exhibiting transverse ribs." Physics of Fluids 18, no. 8 (2006): 087110. https://doi.org/10.1063/1.2336453

[5] Teo, Chiang Juay, and Boo Cheong Khoo. "Analysis of Stokes flow in microchannels with superhydrophobic surfaces containing a periodic array of micro-grooves." Microfluidics and Nanofluidics 7, no. 3 (2008): 353. https://doi.org/10.1007/s10404-008-0387-0

[6] Karatay, Elif, A. Sander Haase, Claas Willem Visser, Chao Sun, Detlef Lohse, Peichun Amy Tsai, and Rob G. H. Lammertink. "Control of slippage with tunable bubble mattresses." Proceedings of the National Academy of Sciences 110, no. 21 (2013): 8422-8426.

https://doi.org/10.1073/pnas.1304403110

[7] Enright, Ryan, Marc Hodes, Todd Salamon, and Yuri Muzychka. "Isoflux Nusselt Number and Slip Length Formulae for Superhydrophobic Microchannels." Journal of Heat Transfer 136, no. 1 (2013). https://doi.org/10.1115/1.4024837

[8] Maynes, D., B. W. Webb, and J. Davies. "Thermal Transport in a Microchannel Exhibiting Ultrahydrophobic Microribs Maintained at Constant Temperature." Journal of Heat Transfer 130, no. 2 (2008).

https://doi.org/10.1115/1.2789715

[9] Gould, Paula. "Smart, clean surfaces." Materials Today 6, no. 11 (2003): 44-48.

https://doi.org/10.1016/S1369-7021(03)01131-3

[10] Enright, Ryan, Nenad Miljkovic, Ahmed Al-Obeidi, Carl V. Thompson, and Evelyn N. Wang. "Condensation on Superhydrophobic Surfaces: The Role of Local Energy Barriers and Structure Length Scale." Langmuir 28, no. 40 (2012): 14424-14432. https://doi.org/10.1021/la302599n

[11] Yu, Kok Hwa, Chiang Juay Teo, and Boo Cheong Khoo. "Linear stability of pressure-driven flow over longitudinal superhydrophobic grooves." Physics of Fluids 28, no. 2 (2016): 022001. https://doi.org/10.1063/1.4940336

[12] Duan, Zhipeng, and Y. S. Muzychka. "Slip Flow in the Hydrodynamic Entrance Region of Circular and Noncircular Microchannels." Journal of Fluids Engineering 132, no. 1 (2009). https://doi.org/10.1115/1.4000692

[13] Ferrás, L. L., A. M. Afonso, M. A. Alves, J. M. Nóbrega, and F. T. Pinho. "Development Length in Planar Channel Flows of Newtonian Fluids Under the Influence of Wall Slip." Journal of Fluids Engineering 134, no. 10 (2012). https://doi.org/10.1115/1.4007383

[14] Haase, A. Sander, Jeffery A. Wood, Lisette M. J. Sprakel, and Rob G. H. Lammertink. "Inelastic non-Newtonian flow over heterogeneously slippery surfaces." Physical Review E 95, no. 2 (2017): 023105. https://doi.org/10.1103/PhysRevE.95.023105

[15] Muzychka, Y. S., and R. Enright. "Numerical Simulation and Modeling of Laminar Developing Flow in Channels and Tubes With Slip." Journal of Fluids Engineering 135, no. 10 (2013). https://doi.org/10.1115/1.4024808

[16] Philippou, Maria, Zacharias Kountouriotis, and Georgios C. Georgiou. "Viscoplastic flow development in tubes and channels with wall slip." Journal of Non-Newtonian Fluid Mechanics 234, no. (2016):69-81.

https://doi.org/10.1016/j.jnnfm.2016.04.008 
[17] Kountouriotis, Zacharias, Maria Philippou, and Georgios C. Georgiou. "Development lengths in Newtonian Poiseuille flows with wall slip." Applied Mathematics and Computation 291, no. (2016): 98-114.

https://doi.org/10.1016/j.amc.2016.06.041

[18] Yu, Kok Hwa, Yan Xu Tan, Mohd Sharizal Abdul Aziz, Yew Heng Teoh, and Mohd Zulkifly Abdullah. "The Developing Plane Channel Flow over Water-Repellent Surface Containing Transverse Grooves and Ribs." Jounal of Advanced Research in Fluid Mechanics and Thermal Sciences 45, no. 1 (2018): 141-148.

[19] Yu, Kok Hwa, Yew Heng Teoh, Mohd Azmi Ismail, Chih Fang Lee, and Farzad Ismail. "Numerical Investigation on the Influence of Gas Area Fraction on Developing Flow in a Pipe Containing Superhydrophobic Transverse Grooves." Jounal of Advanced Research in Fluid Mechanics and Thermal Sciences 45, no. 1 (2018): 109-115.

[20] Lee, Ming Wei, Kok Hwa Yu, Yew Heng Teoh, Han Wei Lee, and Mohd Azmi Ismail. "Developing Flow of Power-Law Fluids in Circular Tube Having Superhydrophobic Transverse Grooves." Journal of Advanced Research in Fluid Mechanics and Thermal Sciences 56, no. 1 (2019): 1-9.

[21] Durst, F., S. Ray, B. Ünsal, and O. A. Bayoumi. "The Development Lengths of Laminar Pipe and Channel Flows." Journal of Fluids Engineering 127, no. 6 (2005): 1154-1160. https://doi.org/10.1115/1.2063088

[22] Poole, R. J., and B. S. Ridley. "Development-Length Requirements for Fully Developed Laminar Pipe Flow of Inelastic Non-Newtonian Liquids." Journal of Fluids Engineering 129, no. 10 (2007): 1281-1287.

https://doi.org/10.1115/1.2776969 